\title{
Aprendizagem da Empatia na Relação Médico-Paciente: um Olhar Qualitativo entre Estudantes do Internato de Escolas Médicas do Nordeste do Brasil
}

\section{PALAVRAS-CHAVE \\ - Empatia. \\ - Relação médico-paciente. \\ - Ensino médico.}

\author{
Empathy Learning in the Medical-Patient \\ Relationship: a Qualitative Look Among \\ Internship Students of Medical Schools in the \\ Northeast of Brazil
}

Nildo Alves Batista ${ }^{I}$ Simone Schwartz Lessa ${ }^{I I}$
${ }^{I}$ Universidade Federal de São Paulo, São Paulo, São Paulo, Brasil.

"Universidade Estadual de Ciências da Saúde de Alagoas, Maceió, Alagoas, Brasil e Centro Universitário Tiradentes, Maceió, Alagoas, Brasil. 


\section{KEY-WORDS}

- Empathy.

- Physician-patient relationship.

- Medical teaching.

Aceito em: 11/9/19

\footnotetext{
Recebido em: 2/8/19
}

ABSTRACT

Empathy is an attribute of the clinical act, of fundamental importance for the establishment of a good doctor-patient relationship. Medical undergraduate courses should guide the students' training, with the purpose of developing skills that favor the qualification of a decisive professional and able to understand the importance of teamwork. This study aims to analyze the learning of empathy in undergraduate students of medical courses from public universities in the Northeast of Brazil, which are in their last year of medical internship. In order to do so, we sought to understand these students' empathy conceptions, to know how the learning for an empathic attitude during medical training occurred and to identify how to improve this learning. A qualitative methodology was used, and focal groups were used with students from two different universities, whose speeches were submitted to a thematic analysis, which tried to apprehend the conceptions of empathy, its learning during undergraduate school and the suggestions for the improvement of its teaching / learning. The results pointed out that students do not recognize or identify relevant teaching moments during their training in relation to the learning of empathy and, when mentioned, they associate it more to theory than to medical practice. They emphasize that the methodology used is not very stimulating and that teachers lack training. They also request an institutional look at their mental health. There were no significant differences between the gender and the age of the students regarding the empathic disposition. It is concluded that this research confirmed the initial assumption: that the medical undergraduate course, in general, has not adequately prepared students for the practice of empathy in the doctorpatient relationship. For these students, the "capacity to put oneself in the other's shoes" stands out as difficult, thus strengthening the need for a more careful look at the training scenario in order to intervene more adequately to increase the learning of the empathy component.

\section{INTRODUÇÃO}

Pensar a "relação médico-paciente" é refletir sobre a prática cotidiana do médico e a maneira como se dá a formação desse profissional ao longo dos tempos. É pensar o processo de interação humana que deve ser a base dessa prática em suas dimensões técnicas, humanísticas e éticas.

Estudos dessa temática concluem que, apesar da existência de uma eficácia terapêutica cada vez mais refinada e de um aparato tecnológico bem mais desenvolvido, fatores diversos causaram a diminuição do vínculo médico-paciente ao longo do último século ${ }^{1}$.

O estabelecimento de uma boa relação médico-paciente se constitui em um fator essencial para o cuidado em saúde, em que a empatia é um dos pilares dessa relação. O estabelecimento de relações empáticas implica não só uma maior satisfação dos pacientes, mas também dos próprios profissionais de saúde. Fortalece os vínculos, promove uma melhor adesão ao tratamento e diminui as queixas e os processos jurídicos contra o profissional ${ }^{2}$.

Stepien e Baernstein ${ }^{7}$ consideram a empatia uma habilidade de caráter multidimensional que possibilita perceber e en- tender o sentimento de alguém, de acordo com a perspectiva dele. Para esses autores, a empatia é considerada uma virtude, no entanto, para outros estudiosos, trata-se de um processo intelectual e de um comportamento ou uma atitude que podem ser aprendidos e aprimorados durante a vida. Caso seja considerada uma habilidade, está atrelada à inteligência emocional e passa a ser um dos domínios das habilidades de comunicação.

Os cursos de graduação em Medicina devem orientar a formação com o propósito de desenvolver competências que favoreçam a capacitação de um profissional crítico e apto a entender a importância do trabalho em equipe, com vistas à integralidade na assistência prestada, pautada na prática humanizada e respeitosa. São princípios contidos nas Diretrizes Curriculares Nacionais (DCN) para os cursos de Medicina, homologadas em $2001^{4}$ e revistas nas DCN de junho de $2014^{5}$.

As DCN são norteadoras do processo educacional vigente que se evidencia pelas experiências inovadoras em educação, por algumas reformas curriculares em diversas escolas médicas, pela iniciativa da instalação da cultura avaliativa e por uma crescente produção acadêmica na área da educação médica ${ }^{6}$. 
Raramente, os cursos propiciam momentos para uma abordagem relativa à compreensão das expectativas do paciente e da relação médico-paciente, inerentes a essa realidade ${ }^{7}$.

Para realizar um cuidado de qualidade, é necessário o desenvolvimento de habilidades e de competências que ajudem o profissional não só a conhecer a doença do paciente, como também os aspectos subjetivos do "estar doente" e o sofrimento que essa condição permeia. Uma conduta mais empática favorece a acurácia do diagnóstico, melhora a efetividade das intervenções terapêuticas por meio de uma maior adesão ao tratamento e aumenta a satisfação na relação médico-paciente . $^{8}$

Hojat e LaNoue ${ }^{9}$ destacam que atitudes empáticas tornam o profissional mais preocupado com a compreensão do tipo e da qualidade das queixas, como também das vivências do paciente. Contudo, um profissional com atitudes simpáticas está mais preocupado com o sentimento relativo à intensidade e à quantidade desses sentimentos.

Neste estudo, procura-se entender em que patamar se encontra o desenvolvimento da empatia entre os estudantes de medicina que já cursaram a maior parte do seu currículo de formação e que estarão, em breve, aptos a exercer a profissão. A relevância dessa investigação se justifica quando se avalia a empatia por meio do olhar de quem está em formação e se compreende essa habilidade como um importante componente da vida em sociedade, pela influência positiva nas tomadas de decisões e no cuidado com o outro. Outrossim, espera-se contribuir para o aprimoramento da formação do futuro profissional no contexto da educação médica, no que concerne ao ensino durante a graduação.

\section{MÉTODO}

Este estudo é parte de uma pesquisa mais abrangente desenvolvida pela autora para obtenção do título de doutora pela Universidade Federal de São Paulo (Unifesp). Este trabalho foi submetido ao Comitê de Ética da Unifesp e aprovado por ele, conforme Parecer n⿳0 623-050/2014.

Foi realizada uma análise temática das falas de dois grupos focais, com o total de 15 alunos do último período do internato médico: um da Universidade Federal de Alagoas (Ufal) e outro da Universidade Estadual de Ciências da Saúde de Alagoas (Uncisal).

Nos dois encontros, informou-se aos estudantes que as falas seriam gravadas e que poderiam responder livremente às três questões norteadoras da pesquisa:

- Qual é a sua concepção/seu entendimento de empatia na relação médico-paciente?

- Como você vê o processo de ensino-aprendizagem da empatia durante a graduação médica?
- Quais são as suas sugestões para o aprimoramento desse ensino?

Como não existiram diferenças significativas nas falas dos dois grupos, não foram necessárias análises separadas.

Com base nas falas, destacaram-se as unidades de contexto (UC) para cada um dos núcleos direcionadores, das quais emergiram as unidades de registro (UR). Na Tabela 1, encontra-se demonstrada a quantidade de UR, identificada nas falas dos internos, de acordo com os núcleos direcionadores.

\section{RESULTADOS E DISCUSSÃO}

Os resultados se referem à concepção de empatia, de como ocorre o aprendizado da empatia, bem como às sugestões feitas pelos alunos para o aprimoramento do aprendizado da empatia na graduação.

A Tabela 1 apresenta a quantidade de vezes em que cada um dos núcleos direcionadores foi comentado.

\section{TABELA 1}

\begin{tabular}{|c|c|c|}
\hline \multirow[t]{2}{*}{ NÚCLEOS DIRECIONADORES } & \multicolumn{2}{|c|}{ UR } \\
\hline & No & $\%$ \\
\hline Concepção de empatia na relação médico-paciente & 87 & 38,8 \\
\hline $\begin{array}{l}\text { O ensino/aprendizagem da empatia na graduação } \\
\text { médica }\end{array}$ & 76 & 33,9 \\
\hline $\begin{array}{l}\text { Sugestões para aprimoramento do ensino na } \\
\text { graduação }\end{array}$ & 61 & 27,3 \\
\hline TOTAL & 224 & 100 \\
\hline
\end{tabular}

Fonte: Elaborado pelos autores.

\section{CONCEPÇÃO DE EMPATIA PELOS ESTUDANTES}

A análise temática desse núcleo direcionador permitiu a identificação de 38 UC com 87 UR. Dessas UR emergiram as categorias explicitadas a seguir.

\section{QUADRo 1}

\section{Núcleo direcionador 1: concepção de empatia}

\section{Categorias}

1. Atitude de ajuda ao outro.

2. Postura de colocar-se no lugar do outro.

3. Atitude de compaixão perante o paciente.

4. Componente fundamental da relação médico-paciente.

5. Processo que envolve variáveis relacionadas ao comportamento do paciente e da família.

6. Processo de construção diária na relação médico-paciente.

7. Processo que demanda limites no envolvimento profissional. Fonte: Quadro elaborado pelos autores. 
Os internos referem-se à empatia como uma atitude de ajuda ao outro: "E quando não existem soluções, a gente luta para pelo menos confortar o paciente com atenção".

A concepção dos estudantes quanto à questão "empatia como ajuda ao paciente" mostra-se um tanto reducionista em relação aos conceitos predominantes na literatura. Como se salientou no referencial teórico, a empatia envolve diferentes domínios, como o cognitivo, o afetivo e o emocional ${ }^{10}$. Dessa maneira, como sinalizam Stepien e Baernstein 7 , a empatia é um dos fatores que possibilitam perceber e entender o sentimento de alguém na perspectiva deste, o que não se restringe a uma atitude de ajuda no sentido do conforto, da atenção e do cuidado, como referem os estudantes.

Costa, Poles e Silva ${ }^{11}$, em estudo com estudantes de medicina e enfermagem sobre formação em cuidados paliativos, relatam que, após a vivência com pacientes que necessitavam desses cuidados, os estudantes também demonstraram sentimentos de preocupação com eles, p que envolvia atitudes de ajuda emocional, conforto e respeito à dignidade.

Nas falas dos alunos, aflora também a empatia como postura de colocar-se no lugar do outro: "a empatia envolve a possibilidade de se colocar no lugar do outro, de tentar perceber como o paciente se sente".

Percebe-se que os estudantes entendem esse componente da empatia apontado na literatura. Considerada como um dos componentes afetivos da empatia, a capacidade de colocar-se no lugar do outro não implica experimentar os mesmos sentimentos do paciente, mas entender o que ele está sentindo e que isso pode ser permeado por um sentimento de afeto ${ }^{12}$.

Os estudantes, no entanto, reconhecem as dificuldades de colocar em prática esse componente da empatia: "nem sempre estamos preparados para lidar com o sofrimento do outro, com a dor do outro, mas precisamos aprender a nos colocar no lugar dele".

Araújo e Peixinho ${ }^{13}$, nos relatos dos diários de campo de estudantes de medicina da disciplina de propedêutica da Universidade Federal da Bahia (UFBA), encontraram falas semelhantes, nas quais alguns alunos relataram o desconforto em se colocar no lugar do paciente por não se imaginarem naquela situação.

Uma atitude de compaixão perante o paciente é também uma concepção de empatia apontada pelos estudantes neste estudo: "Compaixão para mim é um sentimento que sinto quando estou com um paciente e sinto pena de sua situação".

A compaixão é vista pelos internos como uma resposta subjetiva que emerge da relação obtida quando acontece a afinidade com o paciente. Hojat ${ }^{10}$ consideram que o limite desse componente é tênue e pode implicar um desgaste emocional muito forte para o médico, impedindo-o de conduzir adequadamente o cuidado esperado ao paciente.

Os estudantes ainda concebem a compaixão como um atributo intrínseco, nato do ser humano: "eu acho que ter compaixão é muito mais natural do que eu me colocar no lugar do outro".

Em estudo com docentes de medicina da Universidade Estadual de Campinas (Unicamp), Costa e Azevedo ${ }^{14}$ também obtiveram alguns relatos em que esse componente emocional da empatia é intrínseco ao indivíduo, perpassa pela educação regular, pela família e pelos aspectos culturais. Paro, Gallotti, Tibério, Pinto e Martins ${ }^{15}$ relatam também esse sentimento entre estudantes brasileiros e destacam a religiosidade como um fator determinante para esse sentimento de compadecimento, destacando a religião católica, ainda predominante na população brasileira, como indutora do sentimento de caridade e compaixão.

A empatia é reconhecida pelos estudantes como um componente fundamental da relação médico-paciente, sendo entendida como um processo de construção diária: "a empatia é essencial para a relação médico-paciente, mas tudo isso é construído no dia a dia".

Observa-se que reconhecem a importância da empatia para o estabelecimento de uma boa relação médico-paciente, o que está de acordo com a literatura que enfatiza e reconhece esse binômio empatia - Relação médico paciente - como fundamental para o ato médico.

Nesta investigação, muitos estudantes concebem a atitude empática como um desafio na relação que esbarra em muitas dificuldades: "as situações que vivenciamos no dia a dia interferem na relação com os pacientes, como também na empatia [...]".

Outra categoria que emerge das falas é o entendimento de empatia como um processo que demanda limites no envolvimento profissional, especialmente no aspecto emocional: "devo ter empatia, mas não posso me envolver muito com o paciente, senão fica difícil para minha conduta".

Esse ponto ressaltado pelos internos é também considerado na literatura. Hojat et al. $^{8}$ comentam que, uma vez em excesso, a empatia pode dificultar a acurácia diagnóstica e a condução da terapêutica.

Martins ${ }^{16}$ assinala que a atividade médica no ato de cuidar é fonte de gratificação e também de estresse. $\mathrm{O}$ autor considera como fatores gratificantes: diagnosticar e tratar corretamente, prevenir agravos, aconselhar, curar e receber reconhecimento. São fatores estressantes: ter contato frequente com a dor e o sofrimento, lidar com as expectativas dos pacientes e familiares e com as limitações do conhecimento científico e entender os limites emocionais da relação médico-paciente e da empatia. 


\section{O ENSINO/APRENDIZAGEM DA EMPATIA NA GRADUAÇÃO MÉDICA}

A análise temática desse núcleo permitiu a identificação de 28 UC com 76 UR. Dessas UR emergiram as categorias apresentadas a seguir.

\section{QUADRo 2}

Núcleo direcionador 2: o processo de ensino/

aprendizagem da empatia na graduação médica

Categorias

1. Deficiência na aprendizagem da empatia durante a graduação decorrente da elitização do curso.

2. Deficiência na aprendizagem da empatia decorrente de metodologias pouco atrativas e desarticulação teoria-prática.

3. Capacitação insuficiente dos docentes para o ensino da empatia.

4. Pouco preparo dos estudantes para atitudes empáticas, evidenciado pela insegurança ao final do curso.

Fonte: Quadro elaborado pelos autores.

Na fala dos internos, existe uma deficiência na aprendizagem da empatia durante a graduação decorrente da elitização do curso. Os estudantes entendem que a aprendizagem da empatia é influenciada por isso, e, muitas vezes, o interno, quando em contato com pacientes mais carentes, passa por um "choque de realidade": "A minha realidade não é essa, aí você se vê em uma realidade completamente diferente da sua, e não sabe como lidar".

Os estudantes relatam que o curso médico é diferenciado e, muitas vezes, considerado elitizado. Isso causa neles uma dificuldade no reconhecimento de outras realidades para as quais não estão preparados, o que prejudica a aprendizagem e o desenvolvimento de habilidades relacionadas com a empatia.

A literatura assinala que a busca pelo curso de medicina ainda está em parte atrelada ao reconhecimento social e ao retorno financeiro, o que, na visão de Grosseman e Stoll ${ }^{18}$, coloca o estudante diante de uma realidade desconhecida por ele, dificultando o processo de aprendizagem da empatia.

Nas falas dos internos, emerge também uma deficiência na aprendizagem da empatia durante o curso, em virtude da utilização de metodologias de ensino pouco atrativas para essa finalidade, não despertando no aluno o interesse pelo tema: "acho que, quando temos essas aulas de saúde coletiva que fala em empatia no começo do curso, não damos valor, não são atrativas [...]".

As metodologias de ensino usadas pelos professores e a consequente deficiência na aprendizagem da empatia na graduação apontada pelos estudantes estão de acordo com o posicionamento de autores como Grosseman e Stoll ${ }^{18}$ que afirmam que essa é uma das razões para a pouca efetividade no processo de construção de conhecimentos. As autoras salientam que a apreensão dessa atitude é, muitas vezes, desenvolvida em disciplinas isoladas e desconectadas da prática.
Segundo Lima ${ }^{19}$, para tornar uma aprendizagem significativa, é necessário que os estudantes deem sentido aos conteúdos repassados e entendam sua aplicabilidade às situações cotidianas que lhes dizem respeito.

Entre outros aspectos, os internos afirmam que muitas vezes ocorre uma desarticulação teoria-prática no ensino da empatia. Consideram que há distância teórico-prática, o que dificulta a aprendizagem e a consequente prática da empatia: “Em relação aos professores, na vida acadêmica, até o quarto ano no caso, a gente vê a presença deles, vemos a teoria na sala de aula [...] é falado, mas não temos prática".

Especificam que existe um descompasso entre a postura prática do professor e o que é ensinado em relação à empatia. Destacam ainda que o aprendizado ocorre, igualmente, no contato com outros médicos não docentes: "A gente acompanha pouco os professores e nem sempre eles agem como ensinam".

Autores como Rossi e Batista ${ }^{20}$ apontam que o aprendizado da empatia na graduação acontece para além do currículo regular dos cursos. Esse aprendizado é também ressaltado nas falas dos estudantes, caracterizando o chamado "currículo oculto", que está relatado na literatura em todos os momentos de aprendizagem da graduação, podendo influenciar positiva ou negativamente a formação dos alunos.

Outro aspecto relatado nas falas dos alunos está relacionado à capacitação insuficiente dos docentes para o ensino da empatia: “infelizmente nós temos pouquíssimos preceptores, pouquíssimas pessoas em que podemos confiar em nosso dia, tirar dúvidas".

A docência no ensino superior, em particular no curso médico, geralmente carece de capacitação do professor, tanto para o ensino da empatia e da relação médico-paciente como para o estabelecimento de uma boa relação professor-aluno. Vieira ${ }^{21}$ e Perim et al. ${ }^{22}$ destacam que o corpo docente dos cursos de medicina é composto essencialmente por médicos de diferentes especialidades que, em sua maioria, não possuem formação específica para exercer a atividade docente.

Os estudantes referem ainda a pouca responsabilidade institucional quanto à avaliação do ensino, o que repercute no ensino da empatia: "Neste quesito eu acho que a universidade tem que ter a sua responsabilidade no ensino, não vejo preocupação em avaliar como aprendemos".

Destacam que a universidade não se compromete, de forma geral, com o processo de aprendizagem, e isso consequentemente reflete na aprendizagem da empatia. Perim et al. ${ }^{22}$, ao analisarem 28 escolas médicas brasileiras quanto à autoavaliação, destacam deficiência no acompanhamento da formação docente, tanto nas atividades pedagógicas e técnico-científicas quanto nas gerenciais. 
Freitas, Sordi e Malavasi ${ }^{23}$, que estudaram a avaliação educacional, ressaltam que o processo pedagógico vigente ainda situa a avaliação como uma etapa final do processo de aprendizagem e não como um processo contínuo que deve caminhar lado a lado com os objetivos pedagógicos institucionais e dos cursos.

Para Taquette, Stella, Costa e Alvarenga ${ }^{24}$, as falhas no desempenho educacional não são fáceis de ser percebidas e medidas, e o processo avaliativo interno não é uma prática habitual na maioria das instituições, o que torna as mudanças curriculares trabalhosas e difíceis.

Por fim, a análise temática evidenciou uma sensação de despreparo dos estudantes para atitudes empáticas, evidenciado pela insegurança no final do curso. Nesse sentido, chamam a atenção o sentimento de "angústia" dos internos no final do curso e o reconhecimento de dificuldades para enfrentar a vida profissional: "eu estou morrendo de medo de sair com meu carimbo. [...] Gente, eu queria mais tempo aqui dentro".

Para os estudantes participantes da pesquisa, esse sentimento angustiante de "ficar perdido" está patente no internato. Paro ${ }^{26}$ adverte que essa "angústia pessoal" provoca no estudante um sentimento de desconforto e ansiedade em face da dificuldade do outro, uma vez que ele não sabe sequer lidar com sua própria dificuldade.

Millan, Marco, Rossi e Arruda ${ }^{25}$ acrescentam que isso gera decepção e sensação de impotência, o que irá se contrapor às fantasias idealizadas de cura e salvação da vida humana. Desse modo, a observação do futuro profissional médico fica dirigida às questões que não sabe, não pode e não consegue resolver, e isso reforça os sentimentos de inadequação, inoperância, angustia e culpa.

Ante esse panorama, os internos apresentam sugestões para o aprimoramento do ensino da empatia na graduação. A análise temática desse núcleo permitiu a identificação de 24 UC com 61 UR. Dessas UR emergiram as categorias explicitadas no Quadro 3.

\section{QUADRo 3}

Núcleo direcionador 3: sugestões para o

aprimoramento do ensino da empatia na graduação

Categorias

1. Melhorar o ensino médico como um todo.

2. Valorizar o ensino da empatia durante a graduação.

3. Melhorar o ensino da empatia, diversificando as estratégias de ensino/aprendizagem.

4. Capacitar os docentes/preceptores para o ensino da empatia.

5. Cuidar emocionalmente dos alunos.

Fonte :Quadro elaborado pelos autores.

Das falas dos estudantes resultaram várias sugestões, uma delas é que o ensino médico precisa melhorar não apenas em relação ao ensino da empatia, mas também de maneira geral: "o curso de medicina precisa rever o currículo, precisa humanizar mais, melhorar a metodologia".

As DCN de 2001, atualizadas em 2014, sinalizam mudanças ao proporem novos modelos pedagógicos com mudanças na estruturação curricular e a adoção de metodologias ativas com o objetivo de formar médicos mais críticos, humanizados e engajados socialmente ${ }^{25}$.

Especificamente quanto ao ensino da empatia, salienta-se a necessidade de valorização do ensino dessas habilidades dentro do currículo: "sabemos que precisa muito mais para poder aprender coisas novas. É importante valorizar o ensino da empatia para sair do achismo".

Os alunos indicam a necessidade de inserir o ensino da empatia durante todo o curso de graduação. Referem que os conhecimentos de ética, comunicação e empatia não devem ser desenvolvidos de forma empírica ou a partir de iniciativas isoladas de alguns docentes.

Entende-se que é premente a implantação e implementação de abordagens das habilidades de comunicação, nos currículos de graduação, de modo transversal durante o curso, como também a ênfase dos conteúdos humanísticos. Rios e Sirino ${ }^{27}$ sinalizam que a dificuldade de clarificação do conceito de humanização e a inserção desses conteúdos na graduação, ainda, são superficiais, possuem uma discreta familiarização por parte dos docentes e discentes e são desconsideradas, de certo modo, pelos currículos.

Como foi relatado anteriormente, uma das razões para a deficiência da aprendizagem está relacionada à metodologia utilizada nos cursos. Nesse sentido, os internos sugerem uma diversificação das estratégias metodológicas, de modo a torná-las mais atrativas e favorecer a articulação teórico-prática: "seria muito legal no internato discutir casos clínicos de forma multidisciplinar levando em consideração esse objetivo".

A inserção tardia no currículo de graduação do ensino da empatia e das habilidades de comunicação e a forma como são ministradas essas aulas dificultam o processo de aprendizagem dessas habilidades. Para que uma aprendizagem realmente ocorra, a informação deve ser significativa para o aprendiz e desenvolvida ao longo de toda a formação.

Araújo, Salles, Oliveira e $\operatorname{Costa}^{28}$ preconizam que as estratégias metodológicas para o aprimoramento da aprendizagem da empatia devem criar oportunidades para que o aluno possa exercer da melhor forma possível habilidades que favoreçam uma relação médico-paciente pautada numa visão ético-humanística (aprender a fazer), estimulem uma interação mais próxima entre os próprios estudantes (aprender a viver juntos) e invistam no estímulo à autoconsciência dos alunos (aprender a ser), contribuindo para facilitar a abertura à relação com o outro e o desenvolvimento da empatia perante o paciente. 
A capacitação de docentes e preceptores do internato é outra sugestão que permeia as falas. Os alunos salientam a importância da homogeneidade nas orientações e condutas: "Nós apreendemos mais com os médicos que acompanhamos".

É inegável o poder das atitudes dos docentes sobre os seus alunos. Os estudantes esperam um docente comprometido com a formação, em que o ato de ensinar seja perpassado pelo respeito à autonomia do estudante. O papel do docente é mediar as relações entre as informações, os dados, os problemas e as questões que venham a surgir durante o processo de formação, como sinalizam Batista, Vilela e Batista ${ }^{31}$.

Por fim, os internos sugerem que se faz necessário o cuidado com o "emocional" dos alunos: "não pensei que ia ser tão difícil chegar ao final do curso, passei momentos de muito medo".

A literatura aponta que o curso médico é bastante estressante em virtude de sua extensa carga horária, da necessidade de estudos profundos e do distanciamento das atividades sociais ao qual os alunos são submetidos. Além disso, os primeiros contatos entre aluno e paciente são acompanhados de provas e testes de habilidades práticas que nem sempre fornecem experiências agradáveis. Soma-se a isso o fato de o aluno ver suas expectativas confrontadas pela realidade, a qual nem sempre corresponde ao seu idealismo anterior, gerando sentimentos de angústia e insegurança, como relatado por esses estudantes ${ }^{26}$,

Geralmente, o aparelho formador deixa de considerar essas situações, o que causa, muitas vezes, sentimentos de desamparo, solidão e desespero nos estudantes. Esperidião ${ }^{30}$, no cotidiano da docência, destaca que a formação acadêmica, por estar mais voltada para os aspectos técnicos, não disponibiliza espaços para abordar expectativas, anseios e experiências vivenciados pelos alunos durante sua formação.

\section{CONSIDERAÇÕES FINAIS}

Esta investigação permite observar que os estudantes, no tocante à aprendizagem da empatia, não reconhecem nem identificam momentos de ensino relevantes durante a formação e, quando os mencionam, fazem uma relação mais com a teoria do que com a prática médica.

Ao se referirem à atitude de compaixão, surge a ideia de que a empatia é muito mais uma questão de atitude do que uma habilidade essencial para o ato médico.

A partir de suas experiências, os estudantes, inseridos no internato, expressam sugestões para a melhoria do processo de ensino e aprendizagem da empatia e sinalizam para a importância de se investir na humanização, como integrante da cultura institucional, e na capacitação docente em metodologias ativas que favoreçam o processo de ensino e aprendizagem por meio de atividades estimulantes e criativas.
Percebe-se a necessidade de se firmar um compromisso no qual o ensino da empatia seja transversalmente inserido na aprendizagem das habilidades de comunicação, entendido como essencial para um cuidado de saúde efetivo e que possa ser avaliado em todos os momentos da formação médica.

Fica evidente nas falas dos internos a necessidade de "se observar mais de perto" a saúde mental desses jovens. As instituições de ensino, muito mais que formadoras de profissionais tecnicamente competentes, precisam estimular a formação de mentes respeitosas e éticas que, a priori, existem nesses jovens.

Torna-se clara a premência de repensar as relações professor-aluno, médico-paciente e médico-comunidade, entendendo que, para se tornarem efetivas, precisam ser, mutuamente, satisfatórias. Cabe aos responsáveis pela formação médica considerar a diversidade e a complexidade da subjetividade humana, tanto no processo do ensinar e aprender como no processo de adoecer e curar, e reconhecer a importância do papel exercido pelo médico como mediador desses processos.

\section{REFERÊNCIAS}

1. Brasil, Ministério da Educação. Conselho Nacional de Educação. Câmara de Educação Superior. Resolução nº 04 CNE/CES, de 7 de novembro de 2001. Diretrizes curriculares para o curso de medicina.

2. Brasil, Ministério da Educação. Conselho Nacional de Educação. Câmara de Educação Superior. Resolução nº 03 CNE/CES, de 20 de junho de 2014. Diretrizes curriculares para o curso de medicina.

3. Grosseman S, Patrício ZM. Relação médico-paciente evidências de fragilidades e de possibilidades de sua superação por meio do ensino de habilidades em comunicação. In Marins JJN, Rego S, organizadores. Educação médica: gestão, cuidado, avaliação. São Paulo: Abem, Hucitec; 2011.

4. Loureiro J, Pereira MG, Trancas B, Almeida JMC, Caldas $\mathrm{AC}$, et al. Empatia na relação medico-docente: evolução em alunos do primeiro ano de medicina e contribuição para validação da escala Jefferson em Portugal. Acta Med Port. 2011;431-442.

5. Moreto G, Blasco PG. A erosão da empatia nos estudantes de medicina: um desafio educacional. Moreira Jr, 2012.

6. Perim GL, Abdallall IG, Silvalll HRA, Lampertl JB, Stella RCR, Costa NMSC, et al. Desenvolvimento docente e a formação de médicos. Revista Brasileira de Educação Médica. 2009;33(Supl. 1):70-82.

7. Stepien BS, Baernstein A. Educating for empathy. A Review Kathy A. 1 University of Washington School of Medicine, Seattle, WA, USA; 2006. 
8. Hojat M, Mangione S, Nasca TJ, Rattner S, Erdmann JB, Gonnella JS, et al. An empirical study of decline in empathy in medical school. Medical Education. 2004.

9. Hojat M, LaNoue M. Exploration and confirmation of the latent variable structure of the Jefferson scale of empathy. International Journal of Medical Education. 2014;73-81.

10. Hojat M, Gonnella JS, Nasca TJ, Mangione S, Vergare M, Magee M. Phsysician empathy: definition, components, mensurement, and relationship to gender and speciality. American Journal Psychiatry. 2002.

11. CostaÁP,PolesK,SilvaAE.Formaçãoemcuidadospaliativos: experiência de alunos de medicina e enfermagem. Comunicação Saúde Educação. 2016; 20(59):1041-1052.

12. Falcone EMO, Ferreira MC, Luz RCM, Fernandes CS, Faria CA, D'Augustin JF, et al. Inventário de empatia (LE): desenvolvimento e validação de uma medida brasileira. 200; 321-334.

13. Araújo D, Peixinho AL. Avaliação qualitativa em medicina: experiência em propedêutica médica na UFBA, 2003. Revista Brasileira de Educação Médica. 2006;30(2):20-30.

14. Costa FD, Azevedo RCS. Empatia, relação médico-paciente e formação em medicina: um olhar qualitativo. Rev. Bras. Educ. Méd. 2010; 34(2).

15. Paro HBMS, Gallotti RMD, Tibério IC, Pinto RMC, Martins MA. Brazilian version of the Jefferson Scale of Empathy: psychometric properties and factor analysis. BMC Medical Education. 2012;12:73.

16. Martins MCFN. In De Marco MA, organizador. A face humana da medicina: do modelo biomédico ao modelo biopsicossocial. São Paulo: Casa do Psicólogo; 2003. p. 83-99.

17. Fonseca MJM, Fonseca AC. Cuidados paliativos para idosos na unidade de terapia intensiva: realidade factível. Scientia Medica. 2010;20(4):301-309.

18. Grosseman S, Stoll CI. O ensino-aprendizagem da relação médico-paciente: estudo de caso com estudantes do último semestre do curso de medicina. Revista Brasileira de Educação Médica. 2008;32(3).

19. Lima G. Ensinar e aprender: a construção de um conceito. In Batista NA, Silva SHS. O professor de medicina. São Paulo: Loyola; 2001

20. Rossi PS, Batista N. A. The teaching of communication skills in medical schools - an approach. Interface - Comunicação, Saúde e Educação. 2006;10(19):93-102.

21. Vieira MR. Marcas do processo formativo do professor médico com alta tendência empática. (Dissertação de Mestrado). Santa Maria: Universidade Federal de Santa Maria; 2007.

22. Perim GL, Abdalla IG, Aguilar-da-Silva RH, Lampert JB, Stella RCR, Costa NMSC. Desenvolvimento docente e a formação de médicos. Rev. Bras. Educ. Méd. 2009;33 (Supl. 1): 70-82.

23. Freitas LC, Sordi MRL, Malavasi MMS. Avaliação educacional: caminhando pela contramão, Petrópolis: Vozes; 2017.

24. Taquette SR, Stella R, Costa LM, Alvarenga FB. Currículo paralelo: uma realidade na formação dos estudantes de medicina da Uerj. Abem. 2003; 27(3).

25. Millan LR, Marco OLN, Rossi E, Arruda PCV. O universo psicológico do futuro médico: vocação, vicissitudes e perspectivas. São Paulo: Casa do Psicólogo; 1999.

26. Paro HBMS. Empatia em estudantes de medicina no Brasil: um estudo multicêntrico. (Tese de Doutorado). São Paulo: Universidade de São Paulo; 2013.

27. Rios IC, Sirino CB. A humanização no ensino de graduação em Medicina: o olhar dos estudantes. Rev. Bras. Educ. Méd. 2015;(39):401-409.

28. Araújo DGD, Salles CMC, Oliveira MC, Costa AC. Práticas do eixo humanístico no curso de graduação em medicina da Escola Bahiana de Medicina e Saúde Pública. Caderno Abem. 2014;10.

29. Chinato IB, D'Agostini CL, Marques RR. A relação médico-paciente e a formação de novos médicos: análises de vivências de hospitalização. Rev Bras Med Fam Comunidade. 2012; 7(22):27-34.

30. Esperidião E. A relação professor-aluno e a construção da relação com o paciente. In Gonzales RFY, Rodrigues B. A relação com o paciente: teoria, ensino e prática. São Paulo: Guanabara Koogan; 2003.

31. Batista NA, Vilela R, Batista S. Educação médica no Brasil. São Paulo: Cortez; 2015.

32. Provenzano BC, Machado APG, Rangel MTAS, Aranha RN. A empatia médica e a graduação em medicina. Revista HUPE. 2014;13(4):19-25.

\section{CONTRIBUIÇÃO DOS AUTORES}

Ambos os autores participaram de todas as etapas do estudo.

\section{CONFLITO DE INTERESSES}

Não há conflito de interesse.

\section{ENDEREÇO PARA CORRESPONDÊNCIA}

Simone Schwartz Lessa

Endereço para correspondência: Rua Dr. Júlio Sawer, 68 apto.

504 - Ponta Verde - Maceió/AL

CEP: 57035-390

Email: ss-lessa@hotmail.com 